\title{
MedienPädagogik
}

Themenheft Nr. 27: Tagungsband: Spannungsfelder und blinde Flecken. Medienpädagogik zwischen Emanzipationsanspruch und Diskursvermeidung. Herausgegeben von Sven Kommer, Thorsten Junge und Christiane Rust.

\section{Blinde Flecken und präskriptive Un-schuld Zur Morphologie medienpädagogischer Urteilsformen}

Rainer Leschke

\section{Zusammenfassung}

Die implizite Normativität des eigenen Vorgehens gehört bekanntlich zu den am besten bewahrten Geheimnissen der meisten kulturwissenschaftlichen Projekte. Im allgemeinen kulturwissenschaftlichen Feld mag das noch angehen und als lässliche Schwäche kodiert werden, im Bildungsbereich hingegen ist das geradezu fatal, werden doch Biographien mit Zielvisionen beschwert, deren sie sich - wenn überhaupt - nur noch schwer wieder entledigen können. Diese impliziten Leitmodelle von Medienbildung präformieren daher nicht nur Bildungsprogramme und strapazieren damit kollektive Ressourcen, sie statten Biographien mit kulturellen Leitplanken und Reiseprogrammen aus, die eigentlich durch nichts, wenigstens nicht durch Reflexion gedeckt sind. Am normativen Grund der Medienbildung herrscht mithin ein systematisches Reflexionsdefizit und dass dieses jetzt angegangen werden soll, ist sicherlich nicht zufällig, denn zumindest eines dürfte gegenwärtig Konsens sein: die zunehmende Erschlaffung der normativen Kraft und Verbindlichkeit von für mehr oder minder ewig erachteten Vorstellungen von Subjektivität, Wissen und Bildung. Der zunehmende Verlust der Legitimität und Anerkennung dieser impliziten Normativität setzt Medienpädagogik unter einen enormen Rechtfertigungsstress. Sie erzeugt so jene Spannung, die medienpädagogische Bemühungen verortet zwischen einem kaltschnäuzig blinden ‘Weiter so!〉, das die Scheuklappen nur umso entschlossener ins Gesicht zieht, und einer zaghaften Orientierungslosigkeit, die sich an jeden vorbeiziehenden Strohhalm klammert, wenn sie in jeglichem neuen medientechnologischen Feature zugleich ein neues Menschenmodell heraufziehen sieht. Dabei wird das Menschenbild - im Übrigen ein Bild, und damit eine mediale Kategorie - entweder bewahrpädagogisch konserviert - und insofern verfügt die Medienpädagogik in normativer Hinsicht über einen bewahrpädagogischen Kern, wiewohl sie gerade gegen bewahrpädagogische Vorstellungen angetreten war - oder aber outgesourct und damit an die medientechnologische Findigkeit der Medienindustrie abgegeben. Beide Reaktionsmuster scheinen gleichermassen hilflos zu sein und daher dringend der Reflexion zu bedürfen, zumal es sich um eine Existenzfrage der Medienbildung handelt, die systematisch nicht ohne normativen Hintergrund auskommen und daher nicht in metatheoretische Gefilde entfliehen kann. 


\title{
Blind spots and prescriptive innocence. On the morphology of types of adjudgement in media education
}

\begin{abstract}
The implicit normativity of the own approach belongs, as is generally known, to the best kept secrets of the most research projects in cultural studies. This may still be acceptable in the field of general cultural studies - considered as an inattentively deficiency - whereas in the field of pedagogy, such an implicit premise is almost fatal. Biographies are charged with ideas, which they cannot get rid of that easily - if at all possible. This is why these implicit overall concepts of media education are not only preforming education programmes and thereby straining collective resources - they rather equip biographies with cultural guardrails and travel programmes which are actually covered by nothing, at least not by reflection.

Now and again, at the normative bottom of media education dominates a systematic deficit of reflection and obviously it does not happen accidentally, that this deficit of reflection is about to be tackled now. For at least this might currently be consensus: The increasing atony of the normative force as well as the obligingness of concepts of subjectivity, knowledge and education, which are considered to be more or less eternal.

Due to the increasing loss of legitimacy and acknowledgment of this implicit normativity, media education is put under a vast stress of justification. As a consequence, it is creating that tenseness which is locating any effort in media education between a callous and blind «keep it up!» on the on hand and a tentative disorientation on the other hand, which is clutching at straws while attributing a new concept of man to any new feature of media technology. Thereby, the image of humanity - by the way an image and with that a medial category - is either maintained in a conservative way, which is why media education from a normative point of view inevitably possesses a «bewahrpädagogisch» core, - or alternatively outsourced and hence committed to the technological resourcefulness of media industry. Both reaction patterns seem to be equally helpless and do urgently require reflection, particularly since it is a matter of survival of media education which systematically necessitates a normative background and thus cannot escape to meta-theoretical realms.
\end{abstract}




\section{Glückliche Betriebsunfälle - oder gnädige Katastrophen}

Auch die Medienwissenschaft hatten einen schwierigen Start: Ihr Gründungsvater - Marshall McLuhan - stand lange im Verdacht, ein Scharlatan, ein Pop-Idol, ein verkappter Theologe, ein Idiot', zumindest aber ein ernsthaft Kranker gewesen zu sein, und so fiel es noch ziemlich lange auf die Medienwissenschaften zurück, dass ihre Keimidee von einem spiessigen Narren in die Welt gesetzt worden war. Auf jeden Fall war der Start gründlich missglückt und so recht hat eigentlich bis heute niemand die Urszene der Medienwissenschaft von diesem Makel befreien können. Und selbst der zweite, wesentlich von der Frankfurter Schule verantwortete europäische Anlauf verlief nicht wesentlich glücklicher, führte er doch zu einer marxistisch anmoderierten Medienkritik, die mithilfe einer nahezu militaristisch orthodoxen Terminologie ritualisiert das Mediensystem exekutierte und Medien so gewohnheitsmässig entlarvte wie andere sich die Zähne putzen. Die Akteure erinnern sich für gewöhnlich nur ungern noch an diese Ausfälle ebenso theoretisch devoter und anspruchsloser wie den Objekten gegenüber rigider Kritik, sie wissen nur noch eines: Die Zeiten waren heroisch, denn sie bedeuteten Aufbruch. Sie waren getragen vom Eros des ersten Mals und sie waren folgenreich, nämlich die Urszene einer neuen Disziplin: der Medienwissenschaft. Und wie das mit Urszenen so ist, es zählte der Akt selbst und seine nicht ganz so rühmlichen Bedingungen wurden, sobald die Angelegenheit erst einmal etabliert war, sehr schnell dem Vergessen überantwortet.

Nun mag es mit der Verdrängung der marxistischen Frühgeschichte noch so einigermassen geklappt haben, denn das kollektive Tabu der 1968er hat insgesamt ganz gut funktioniert, McLuhan allerdings ist so laut und so unignorierbar medienpräsent gewesen, dass man ihn nicht einfach verdrängen konnte. Was blieb, war kritische Kritik, also die Auseinandersetzung mit dieser verunglückten Geburt einer Disziplin, und die hat zumindest eines bewirkt: Sie hat einen enormen Legitimationsbedarf generiert. Medienwissenschaftler müssen für gewöhnlich ziemlich gründlich Rechenschaft darüber geben, was und warum sie es tun. Solche verunglückten Starts und Betriebsunfälle² belasten daher die weitere Entwicklung einer Disziplin nicht nur, sie können gelegentlich auch kathartische Effekte ${ }^{3}$ zeitigen, und

1 «Heute hat diese apolitische Avantgarde ihren Bauchredner und Propheten in Marshall McLuhan gefunden, einem Autor, dem zwar alle analytischen Kategorien zum Verständnis gesellschaftlicher Prozesse fehlen, dessen wirre Bücher aber als Sandgrube unbewältigter Beobachtungen an der Bewußtseins-Industrie dienen können. [...] Mehr Interesse verdient vielleicht der berühmteste Satz dieses Marktschreiers: 〈The medium is the message〉. Trotz seiner provozierenden Idiotie verrät er mehr, als sein Urheber weiß. Er stellt den tautologischen Zug der Medienmystik auf das Genaueste bloß: [...]». (Enzensberger 1970, 177f.).

2 So etwa erklärte Ludwig Pfeiffer auf einer Siegener Konferenz zum paradigmatischen Status der Medienwissenschaft den 〈Fehl〉-Start der Anglistik.

3 Wiewohl die Katharsisthese die einzige Medienwirkungshypothese zu sein scheint, die einigermassen zuverlässig ausgeschlossen werden kann. 
so gibt es durchaus noch Anlass zur Hoffnung, dass trotz des missglückten Starts aus der Angelegenheit noch etwas werden könnte.

Warum diese Geschichte? - Aus medienwissenschaftlicher Sicht fallen einem, wenn man sich mit der Medienpädagogik auseinandersetzt, durchaus eine Reihe von Parallelen auf. Denn der Start der Medienpädagogik ist kaum minder vermasselt worden als der der Medienwissenschaften: Eine Disziplin, die je nach Lesart von einem Missverständnis oder aber einem grandiosen wissenschaftlichen Leichtsinn ihren Ausgang nahm, hätte eigentlich das ein oder andere zu erklären. Nun hat sie allerdings diesen Bedarf bis dato offensichtlich noch nicht so nachdrücklich gespürt, dass irgendetwas passiert wäre. Die Phase der Kritik steht also noch aus. Denn offenbar hat der enorme politische Erfolg ${ }^{4}$, der der Medienpädagogik von Anfang an beschieden war, sich anders ausgewirkt, als die fragwürdige Berühmtheit McLuhans; er hat nicht zur Kritik animiert, sondern sich wie Balsam auf die offenen theoretischen Wunden gelegt.

Wenn denn nun partout die blinden Flecken der Medienpädagogik in den Blick genommen und ihre theoretische Besenkammer aufgeräumt werden soll, dann handelt es sich zunächst einmal in einem ziemlich strengen Sinne um eine medienwissenschaftliche Operation, denn blinde Flecken sind in optischen Medien zunächst einmal jener unverfügbare und allenfalls rekonstruierbare technische Ort, an dem das Subjekt sitzt. Zu ihrer Erfassung benötigen blinde Flecken bekanntlich einer reflexiven Operation und einer Veränderung des Standortes des Beobachters. Es geht also um die Reflexion des Ortes, von dem aus gesehen wird. Dann schauen wir uns die Sache einmal von aussen an.

\section{Normative Kalküle}

Medienpädagogik startete, sobald sie sich von ihrer bewahrpädagogischen Frühgeschichte befreit hat, mit enormem Elan. Sie begann genauso mit theoretischem Klamauk wie mit marxistisch-emanzipatorischem Sendungsbewusstsein. Im erregten Überschwang kurz bevorstehender umfassender Emanzipation unterliefen ihr zugleich eine ganze Serie unsäglicher Kategorienfehler: Dieter Baacke verwechselte Performanz und Kompetenz $z^{5}$ und er verstand auch nicht, welche

4 So hat sich die Medienpädagogik nicht nur terminologisch mit der Idee von Medienkompetenz weitgehend durchgesetzt, sondern es haben die Konzepte auch Eingang in die Programme der Landesmedienanstalten gefunden.

5 «Dieser Sprachgebrauch ist zulässig und widerspricht keineswegs dem, was 〈Kompetenz〉 meint; freilich wird eher auf die Performanz-Ebene abgehoben (das konkrete Handeln und Verhalten), während die tieferliegenden Hintergründe weniger zum Vorschein kommen.» (Baacke 1996, 114f.) Selbstverständlich ist dieser Sprachıgebrauchı nicht zulässig, denn er vertauscht schlicht die Bedeutungen von Performanz und Kompetenz (vgl. Chomsky 1965, 3). Zusätzlich wird auch noch ein Kategorienfehler begangen, denn Baacke verwechselt zusätzlich noch einen diskreten Unterschied mit einem Kontinuum («eher»). Im Übrigen ist diese Problematik durchaus bemerkt worden (etwa: Kübler 1996, 12 und Gapski 2001, 55ff.), sie blieb aber erstaunlicherweise vollkommen folgenlos. 
theoriestrategische Funktion ein regulatives Prinzip wie die kommunikative Kompetenz überhaupt hat. Baacke agierte wie ein Grobschmied in einer Uhrmacherwerkstatt ${ }^{6}$ und es schien lange Zeit niemandem aufzufallen. Medienpädagogik fusst so auf dem Treibsand eines theoretisch stimulierten Alltagsverständnisses, das für jeglichen Irrtum offen ist. Es handelte sich um eine Art theoretischer Simulation, die in ihrem epistemischen Status durchaus dem vergleichbar war, was in den Medienwissenschaften Vilém Flusser und Paul Virilio inszeniert hatten. Die Angelegenheit hätte sich zweifellos in kürzester Zeit als der komplette Reinfall erwiesen, der sie theoretisch war, und damit erledigt, wäre da nicht zugleich ein erstaunlich sicheres Gespür für medienpolitische Konstellationen gewesen, auf das die theoretische Konstruktion, die sich Medienkompetenz nannte, enorm erfolgreich reagierte. Medienpädagogik hat also eine medienpolitische Urszene mit gelegentlichen theoretischen Grundierungsversuchen und das dürfte für eine Bestimmung blinder Flecken vergleichsweise bedeutsam zu sein, zumal diese Urszene Folgen hatte.

\section{Medienhistorische Konstellationen}

Die medienpolitische Konstellation, in der die Medienpädagogik so vergleichsweise erfolgreich intervenierte, bestand in einer doppelten Umstellung des Mediensystems: nämlich der Transformation eines weitgehend populärkulturellen Mediensystems in eine sozioökonomische Infrastruktur und der Wandel des klassischen in ein postkonventionelles transversal vernetztes Mediensystem, also das, was gelegentlich unter dem ziemlich missglückten Titel - «Medienkonvergenz» verhandelt wird. Beide Transformationen liefen, wiewohl sie sich vollkommen unterschiedlichen Bedingungszusammenhängen verdanken, weitgehend synchron ab, so dass sie zumeist nicht als eigenständige Prozesse identifiziert wurden.

6 Das gilt nicht nur für Baackes Bezug auf Chomsky, sondern ebenso für den auf Habermas und Luhmann. Die Vorgehensweise scheint damit zur Gewohnheit geworden zu sein: «Medienkompetenz ist, um mit Luhmann zu sprechen, eine systemische Ausdifferenzierung aus diesen Zusammenhängen. Daneben gibt es Berufskompetenz, vielleicht Familienkompetenz, demokratische Kompetenz, ästhetische Kompetenz etc.» (Baacke 1996, 119) Medienkompetenz "als systemische Ausdifferenzierung " macht zumindest systemtheoretisch keinerlei Sinn und die metaphorische Verwendung theoretischer Begrifflichkeiten mag vielleicht in journalistischen Kontexten tolerierbar sein, wissenschaftlich gesehen stellt sie ein enormes Risiko dar, das Baacke eindeutig nicht beherrscht. Baackes Synkretismus als Semi-Theorien produzierendes Prinzip wird auch an Folgendem deutlich: «Es ist die Informationsgesellschaft, die den Modus unseres In-der-Welt-Seins heute bestimmt. Nicht das Proletariat (so Marx), sondern das Kognitariat ist heute bestimmend. Minutiös eingestellte Technologien bauen unsere Massendemokratie (und die Massenmedien) ab; es entsteht vielmehr ein Prozess der Entmassung mit Kommunikationsinhalten, die auf Gruppen, Individuen und bestimmte Kulturen zugeschnitten sind. Unterhaltung, Medien, Konsumartikel und ihr Verkauf, aber auch Arbeitsbedingungen lassen einen entmassten Menschen entstehen, der nicht mehr in erster Linie vor dem Grossen Bruder Staat Angst haben muß [...], sondern vor der Privatwirtschaft, die über seine Kommunikations- und Eingabeprozesse seine Daten kontrollieren und weitergeben kann.» (Baacke 1996, 122) Hier rührt Baacke Marx, Nietzsche und die Kritik am Neoliberalismus zu einer reichlich problematischen Melange zusammen, wobei die Einheit durch den ziemlich schiefen Neologismus «Kognitariat» hergestellt wird, der im Übrigen implizit auf Max Bense verweist (Bense 1965,13ff.). 


\section{Ku1ture11e Produktion \\ Kunstsystem wissenschaft Mediensystem}

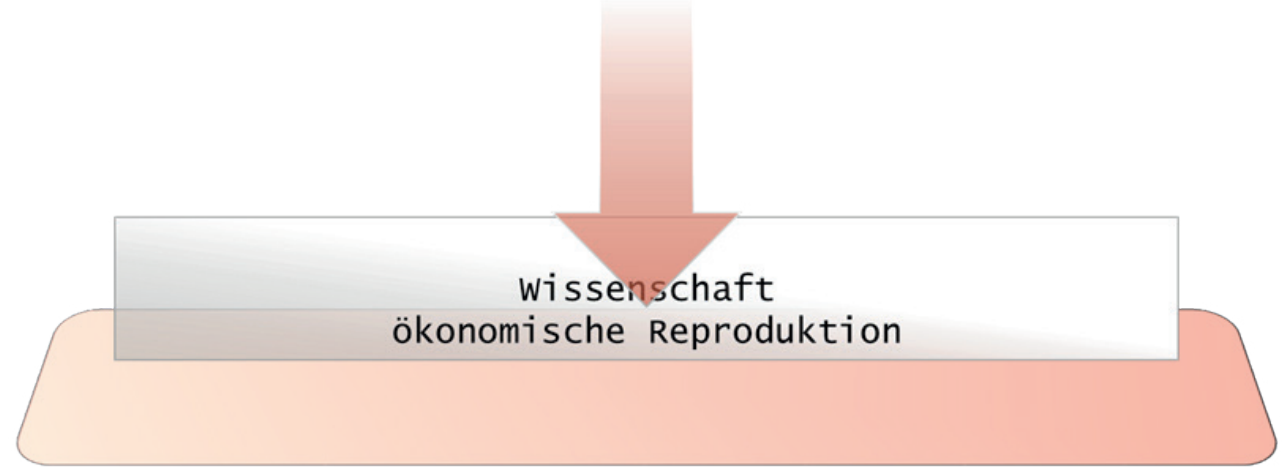

Abb. 1.: Enzensberger identifiziert die Medien nicht mehr als Teil des gesellschaftlichen Überbaus, sondern als zentrales Element der gesellschaftlichen Basis und erkennt damit deren infrastrukturelle Bedeutung an.

Die Transformation eines populärkulturellen Systems in eine Infrastruktur mit universeller sozioökonomischer Relevanz deutete sich zwar schon geraume Zeit an, sie war in ihrer Wucht und vor allem in ihrem Erfassungsgrad jedoch durchaus überraschend. So zählte etwa Enzensberger die Medien immerhin seit Beginn der siebziger Jahre des 20. Jahrhunderts zu den Produktivkräften und hat so ein wenig vorschnell jenen Transfer bereits theoretisch antizipiert, der sich gesamtgesellschaftlich erst Mitte der neunziger Jahre des 20. Jahrhunderts durchsetzen sollte. Als zentrales Movens der gesellschaftlich vorgehaltenen Infrastruktur grundierten Medien seit Mitte der neunziger Jahre nicht mehr nur die gesellschaftlichen Kommunikationsprozesse, sondern zugleich auch immer höhere Anteile der Arbeitsprozesse, der sozialen Interaktion, der Logistik, der technischen Bewegung, des Finanzwesens, der Administration, des kulturellen Konsums und der Freizeit. Als Elemente einer gesellschaftlichen Infrastruktur lassen Medien ihren Nutzer/innen keine Wahl mehr, vielmehr sind sie verbindlich für die Aufrechterhaltung noch der banalsten soziokulturellen und ökonomischen Funktionen. Die Systeme funktionieren nicht nur ausschliesslich medienvermittelt, sondern der Zugang zu ihnen verfügt längst nicht immer auch noch über ein analoges Interface.

Gesellschaftliche Partizipation und Handlungsfähigkeit werden dann automatisch zu einer Frage der Mediennutzung. Wenn bei der Teilnahme jedoch keine Wahl gelassen wird, dann müssen die Zugangsmodalitäten gesellschaftlich geregelt werden: Media-Literacy wird zu einer Erfolgsfrage bei der Implementation einer medialen Infrastruktur und diese wiederum schwingt sich zum Bedingungshorizont ökonomischer Wohlfahrt auf. Medienpädagogik offeriert nun genau das, was 
in dieser Konstellation gebraucht wird, nämlich eine flächendeckende Anpassung von Populationen an eben diese Infrastruktur? ${ }^{7}$. Medienpädagogik heult damit zunächst einmal mit den Wölfen.

Der zweite Transformationsprozess, der Übergang vom klassischen zum postkonventionellen Mediensystem, ist demgegenüber eher eine Frage der Selbstorganisation des Mediensystems denn eine Frage direkter gesellschaftlicher Interessen. Klassische Mediensysteme sind grundsätzlich additiv organisiert gewesen: Es handelt sich um eine historische Ansammlung von in autonomen Dispositiven organisierten Einzelmedien. Das klassische Mediensystem verfügte über einen ziemlich geringen Grad an Binnenorganisation, es handelte sich vielmehr um eine weitgehend kontingente, historische Kollektion von Einzelmedien mit höchst oberflächlicher Vernetzung. Allenfalls eine Art kultureller Integration und Adaptation lässt sich feststellen, die jedoch nie dazu geführt hat, dass die Grenzen der Medien permeabel wurden. Demgegenüber ist das postkonventionelle Mediensystem transversal integriert, d. h., die Grenzen zwischen den Medien sind erodiert und zu einer reinen Stilfrage und damit wählbar geworden. Die Produktionsweise, die Archive, die Plattformen, die Distributionskanäle und Rezeptionsformen sind nicht mehr medienspezifisch organisiert, sondern medienindifferent und damit systematisch integriert. Medienproduktion findet nicht mehr monomedial, sondern bereits standardisiert trimedial - etwa als synchrone Produktion von Rundfunk, Fernsehen und Web-Content - statt. Medienwandel ist nicht mehr materiell und d. h. an Mediendispositive gebunden, sondern schlicht zum Formwandel geworden. Die transversale Integration des Mediensystems hat den Übergang von funktional definierten Kommunikationsinstrumenten und Unterhaltungsmitteln zu einer ebenso generellen wie omnipräsenten Infrastruktur ermöglicht. Dabei kann keineswegs von einer Konvergenz der Medien die Rede sein, sondern die traditionellen Massenmedien bestehen weiterhin als emulierte «Formen` innerhalb einer integrierten medialen Infrastruktur. In dem Moment aber, indem das Mediensystem als kulturelle Infrastruktur angesehen werden muss, ist eine wirksame gesellschaftliche Partizipation ohne elementare mediale Handlungsfähigkeit und Urteilskraft ausgeschlossen.

7 So hat die Kampagne "Schulen ans Netz», die «Digital Divide»-Debatte, Negropontes Idee des 100-Dollar-Laptops vor allem eine Intention, den möglichst vollständigen und schnellen Anschluss aller an die neue Infrastruktur. Medienpädagogik hat damit in durchaus gutem Glauben freiwillig den Job der PR-Abteilungen der IT-Industrie übernommen. Historisch haben sich diese Impulse zumindest in Westeuropa zwischenzeitlich erledigt: «Die Initiative Schulen ans Netz hat ihren Gründungsauftrag erfüllt: Der 1996 gegründete Verein hat daher seine Arbeit zum 31. Dezember 2012 eingestellt.» (http://www.bildungsserver.de/Schulen-ans-Netz-10563.html). 


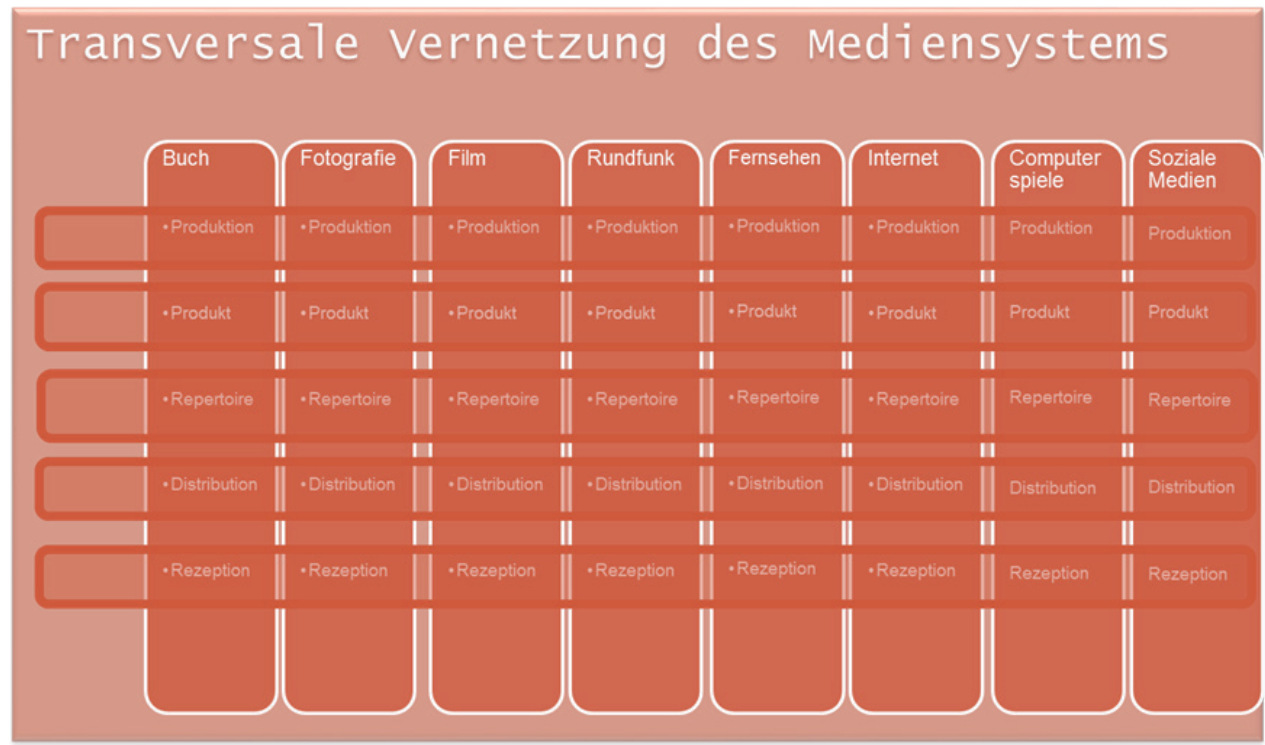

Abb. 2.: Transversale Integration des postkonventionellen Mediensystems und Übergang zu einer kulturellen Infrastruktur.

\section{Enkulturalisierung und Definitionsmacht}

Beide Transformationsprozesse waren grundsätzlicher Art, d. h., sie kennen in dieser Form keine historischen Vorläufer. Beide änderten die gesellschaftliche Vertragsgrundlage, auf der über Medien diskutiert werden konnte, und hatten damit eine epochale Dimension. Beide Transformationsprozesse wurden zunächst einmal behandelt wie all die anderen Medienwechsel auch: d. h., sie wurden mittels der Dialektik von Apokalypse und Erlösung enkulturiert.

Das Charakteristikum solcher Enkulturalisierungsprozesse ist, dass es weniger um die Analyse der Transformationsprozesse als um deren Bewertung geht. Und das hat zur Folge, dass technologisch-infrastrukturelle Prozesse ${ }^{8}$ in kulturelle umcodiert und damit bewertbar gemacht werden. Wenn dann noch der Wandel vor allem als quantitative Zunahme von Medienangeboten und damit als eine Steigerung der Intensität wahrgenommen wird, dann handelt es sich je nach Position um eine Steigerung der Bedrohung oder der Heilserwartung. Die kulturelle Codierung der medienpädagogischen Begründungsdiskurse ist beileibe nicht zufällig, sondern sie hängt mit der Kompatibilität mit bewahrpädagogischen bzw. emanzipatorischen Diskursen zusammen. Die Diskurse der Bewahrpädagogik handelten allesamt vom

8 Der Wechsel vom klassischen zum postkonventionellen Mediensystem ist in der Medienpädagogik unter dem Rubrum Digitalisierung allenfalls an den Rändern angekommen und darin liegt so etwas wie ihre charakteristische Medienvergessenheit. Zugleich gehört Medienpädagogik zwar strukturell zur Begleitmusik der Transformation von Medien, zu einer gesamtgesellschaftlichen Infrastruktur jedoch hat sie auch diesen Transformationsprozess selbst noch nicht wahrgenommen. 
drohenden Verlust kultureller Definitionsmacht und seinen möglichen Folgen und diese Diskurse wurden zumindest topologisch fortgeschrieben, selbst wenn zwischendurch die Wertausstattung mithilfe emanzipatorischer Erwartungen modernisiert wurde. Die kulturelle Codierung von medialen Transformationsprozessen übersetzt einen vergleichsweise offenen technischen Wandel in eine ziemlich geschlossene normative Differenz. Wenn im Zuge einer solchen normativen Komplexitätsreduktion die Seiten gewechselt werden, dann wird zwar die politische Position ausgetauscht, an der systematischen Komplexitätsreduktion ändert sich jedoch noch nicht zwangsläufig etwas. Und auch Baackes historischer Kompromiss zwischen ökonomieaffiner Modernisierung und Medienkritik sucht nur Apokalypse und Erlösung zu versöhnen, er steigert dabei aber weder die Komplexität des normativen Urteils, noch bietet er einen einzigen neuen Wert an.

Nun sind Medien das, was Brecht einmal - «Erfindungen, die nicht bestellt sind» (Brecht 1932, 127) nannte. Sie sind bei ihrem Einbau in eine Kultur zwar technologisch definiert, aber kulturell unterbestimmt, sie sind ein technologischer Möglichkeitsraum. In diese kulturellen Bestimmungsversuche im Kontext von Enkulturationsprozessen neuer Medien waren Pädagogen seit dem Aufstieg des Buchs zum Massenmedium immer schon - wenn auch zumeist bewahrpädagogisch und d. h. abwehrend - involviert. Reguliert werden solche Enkulturalisierungsprozesse stets durch eine Dialektik von Apokalypse und Euphorie, wobei die Medienpädagogik der 1990er Jahre von der bewahrpädagogischen Apokalypse auf die durchaus technikaffine Seite emanzipatorischer Euphorie umschwenkte. Ganz gleich auf welcher Seite man sich in diesen Auseinandersetzungen auch immer befinden mag, das Resultat ist in der Regel eine Form des Mediums, die weder apokalyptischen Befürchtungen noch euphorischen Erwartungen entspricht. Medien werden längst nicht für all das genutzt, was sie technisch können, und die Regeln für den Umgang mit ihnen finden sich nicht in der technischen Betriebsanleitung, sondern sie werden als informelle kulturelle Praktiken etabliert.

Dabei verläuft die Enkulturation von Medien nach einem ausdifferenzierten Phasenschema ab, in dem unterschiedliche soziale, kulturelle und ökonomische Interessen und die zugehörigen sozialen Träger einen kulturellen Kompromiss erzielen, an dessen Ende die kulturelle Definition eines Mediums und der von ihm ermöglichten sozialen und kulturellen Praktiken stehen. Die Dynamik der Enkulturation resultiert aus der zugrundeliegenden Dialektik von Apokalypse und Enthusiasmus, die in den jeweiligen historischen Phasen spezifischen sozialen Trägern zugeordnet werden können, wobei die Medienpädagogik selbst als ein solcher Akteur auftritt. Baacke hat nun in diesem Konflikt die Seiten gewechselt, indem er von der apokalyptischen zur enthusiastischen Position umschwenkte. 


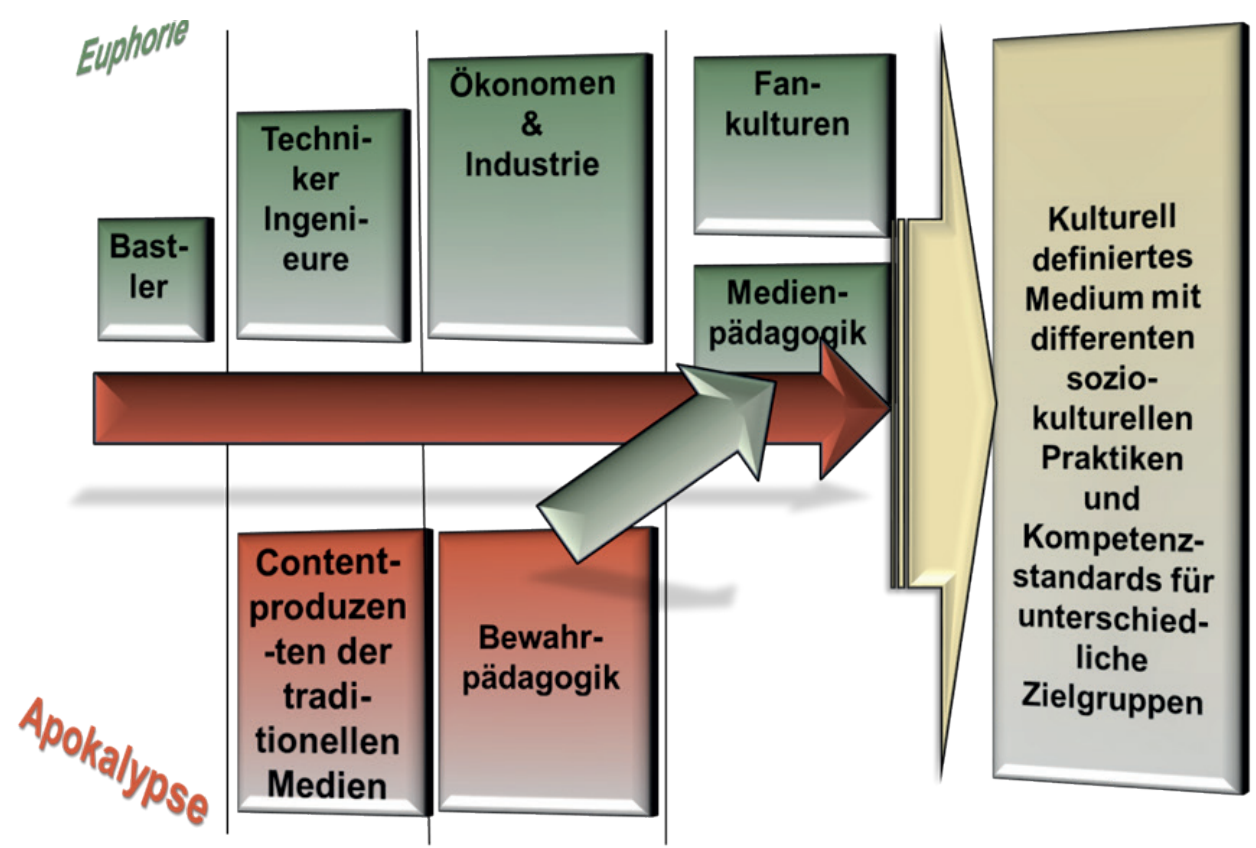

Abb. 3.: Phasen der Enkulturation von Medien und die jeweiligen sozialen Träger und Akteure.

Nun geht es in diesem Prozess um die normative Codierung von Medien und Pädagogik als normative Wissenschaft ist hier immer schon engagiert und betroffen gewesen, das Problem liegt allerdings in der Frage, wo und in welcher Funktion dieses Engagement stattfindet: Der Konflikt von Apokalyptikern und Enthusiasten ist eine politisch-ideologische Auseinandersetzung und Medienpädagogik müsste sich fragen lassen, ob sie sich als Wissenschaft in dieser Auseinandersetzung überhaupt engagieren kann und wie sie sich dann zu positionieren hat. Der Schwenk von der Apokalypse zur Affirmation, den Baacke mithilfe seines frechen historischen Kompromisses von Kulturkritik und technologischer Affinität bewerkstelligte, bewegt sich ausschliesslich innerhalb eines politischen Feldes und d. h. der Auseinandersetzung von Interessen. Medienpädagogik - egal ob als Bewahrpädagogik oder mit einem emanzipatorischen Vorzeichen - hat erstaunlicherweise genau hier ihren Ort gesehen und versucht politisch mitzuspielen. Nur, wenn man auf der Strasse spielt, dann sollte man sich nicht wundern, wenn auch die Regeln der Strasse gelten, d. h., es geht definitiv nicht um Wissenschaft.

Im Übrigen funktioniert in dieser Phase die Medienwissenschaft auch nicht viel anders, sie schwankt genauso zwischen vollkommen überanstrengten Hoffnungen, sollen doch alle Lücken, die Technologien jemals geschlagen, nun mittels der jeweils neuesten Technologie gelöst und in eine schlussendliche Erlösung - Flusser - überführt werden, oder aber der technologisch proliferierten Apokalypse 
einer finalen Unterwerfung des Menschen - Virilio, Bolz - mithilfe von Platinen und Schaltkreisen. Medienwissenschaft operiert also als eine Art Technohermeneutik mit Sinnsetzung. Demgegenüber operiert die Medienpädagogik mit der Komplexitätsreduktion Wertsetzung und d. h. mit einer pädagogischen Technomoral.

\section{Einsetzende Normalität und die Morphologie des medienpädagogischen Urteils}

Nun mag man auf Sinnsetzung zur Not verzichten können, bei normativen Standards fällt das schon erheblich schwerer, zumindest, wenn man einigermassen handlungsfähig bleiben will. Normativer Enthaltsamkeit und vornehm zurückhaltenden Deskriptionen, also dem, was wissenschaftlich in der Regel nach einer solchen Enkulturalisierungsphase Platz greift, gebricht es an der für pädagogische Eingriffe erforderlichen Orientierungsleistung.

Umgekehrt ist die Normativität der Enkulturalisierungsphase strukturell unterkomplex. Überschiessende Interessen liefern überdimensionierte Zielkonstrukte, oder aber sie feiern den Untergang, was beides ausser blinder Zustimmung und ebenso dumpfer Ablehnung keine weiteren Handlungsoptionen generiert. Man changiert so zwischen normativem Überschuss und normativer Unterbestimmtheit. Wenn also der normative Schaum einer weitgehend unverdauten Enkulturalisierungsphase, der zumeist noch nicht einmal selbständig hervorgebracht wurde, sondern sich im Wesentlichen am normativen Inventar der Aufklärung bedient, erst einmal zerfallen ist, dann bleiben vielleicht nicht so sehr blinde Flecken, sondern vor allem weisse Flecken übrig.

Medienwissenschaft kann hier nur bedingt aushelfen. Sie kann jeweils den technisch für die Subjekte vorgesehenen Ort bestimmen und damit die Medieninduziertheit des Subjekts erklären, aber bei der Bewertung ist ziemlich schnell Schluss, kennt sie doch keinen wissenschaftlich auch nur einigermassen verbürgten Ort, von dem aus solche Wertentscheidungen mit einiger Sicherheit zu treffen wären. Zudem sieht die Medieninduziertheit des Subjekts nicht so sehr einen spezifischen Ort, sondern ein Spektrum und gelegentlich auch unterschiedliche Niveaus gleichzeitig vor, d. h., die Aussage operiert mit einer Streubreite oder einem Möglichkeitsraum, aus dem Kulturen dann für spezifische Zielgruppen jeweils einen einigermassen trennscharfen Ort normativ auswählen. Wenn zugleich klar ist, dass es sich dabei mit ziemlicher Sicherheit weder um den Ort der Apokalyptiker noch um den der Euphoriker handelt, dann könnte man versucht sein, sich irgendwie in der Mitte zu einigen. Das Problem ist nur, dass das normative Inventar, mit dem sowohl die Apokalyptiker wie die Enthusiasten operieren, nicht nur medienwissenschaftlich, sondern allein schon historisch vollkommen obsolet ist: es ist 
kulturkonservativ im denkbar schlechten Sinne und liefert damit auch nicht den Stoff für eine auch nur halbwegs akzeptable Mitte.

Insofern schwankt die Angelegenheit zwischen obsoleten Eindeutigkeiten und normativer Abstinenz, zwischen den Idolen der Aufklärung und empirischer Indifferenz. Nun könnte man andererseits das Ganze auch schlicht der Strasse überlassen, d.h. medialen Praktiken, die entstehen, ohne dass sie sich grossartigen formalen Bildungsprozessen verdanken. Die Intuitivität von Benutzerinterfaces legt nahe, dass zumindest die Industrie ihre Hoffnungen auf genau solche Praktiken legt und die Cultural Studies haben das Ganze dann ja auch mit der nötigen Begeisterung versehen. Nur sind die autopoietisch emergierenden medialen Praktiken, wie man am Beispiel von Fankulturen recht gut illustrieren kann, ganz anders, als Benjamin sich das einst noch erhoffte, sie sind nämlich systematisch beschränkt und d. h., man würde, setzte man auf sie, neuen blinden Flecken, ja ganzen blinden Feldern Vorschub leisten. Die Alternativen sind nicht nur aus medienwissenschaftlicher Perspektive nicht wirklich überzeugend: Man könnte nur wahlweise den unterkomplex gewordenen Modellen der Aufklärung, einer grundsätzlichen Handlungs- und Wertabstinenz oder aber den Fankulturen und damit der Industrie das Feld überlassen.

Wenn man meint, dass es Gründe geben könnte, den spontan emergierenden medialen Praktiken und damit dem vereinten Druck von Medienindustrie, Fankulturen und Cultural Studies etwas entgegen zu setzen, dann plädiert man zugleich für einen normativen Eingriff. Und dabei handelt es sich dann für die Medienpädagogik definitiv um einen blinden Fleck, können doch die normativen Setzungen der Enkulturalisierungsphase nicht einfach fortgeschrieben werden, selbst wenn das durch eine resolute Tonlage und den Verweis auf politische Bedeutsamkeit gelegentlich versucht wird.

Von Seiten der Medienwissenschaft können der mediale Möglichkeitsraum bestimmt und die Morphologie des moralischen Urteils analysiert werden, wodurch man eine Art Rahmen hätte, in dem man sich normativ sinnvoll bewegen könnte. Zugleich wären damit die Orte, an denen normative Entscheidungen anstehen, und insofern auch der Handlungsraum der Medienpädagogik identifiziert. Dass dabei die normativen Entscheidungen auf den medialen Möglichkeitsraum zurück zu projizieren und damit medienwissenschaftlich zu kontrollieren sind, versteht sich von selbst, wenigstens wenn man nicht vollständig illusionären Vorstellungen über Medienpraktiken nachhängen will, die sich professionellen Standards verdanken und mithin kaum das Ziel allgemeiner pädagogischer Bemühungen darstellen können. 


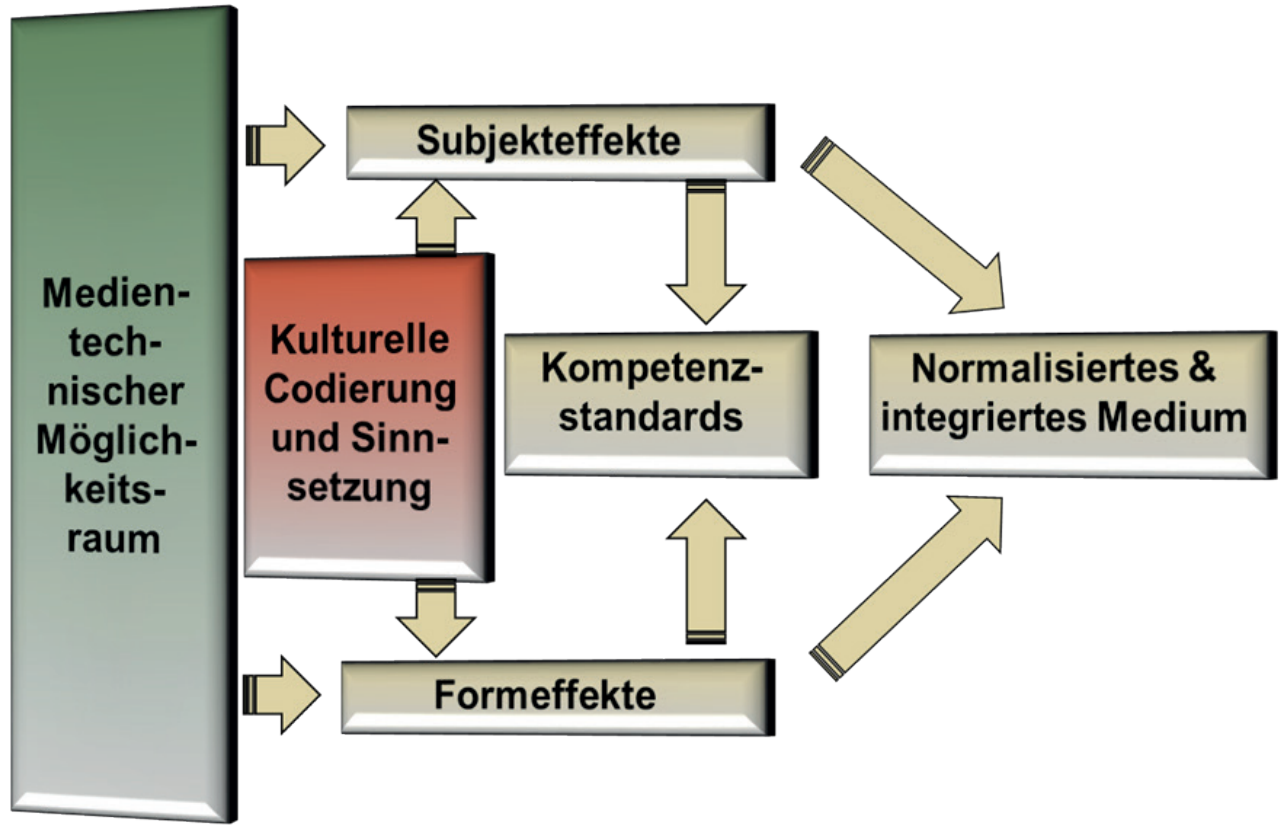

Abb. 4.: Funktionale Ebenen einer medienwissenschaftlich und pädagogisch begleiteten medialen Enkulturation.

Nun sind Fragen der Normsetzung in jedem Fall heikel, sie sind wissenschaftlich prinzipiell nicht abzuleiten oder zu legitimieren - die philosophische Ethik singt seit Jahrhunderten ein Lied davon - und d. h., die Entscheidungen lassen sich solcherart auch nicht rückversichern. Irgendwelche moralischen Glaubenssätze erweisen sich nicht nur in der Regel als strukturell unterkomplex und damit unangemessen, man kann sie vor allem genauso gut glauben wie auch es bleiben lassen. Und der Streit zwischen unterschiedlichen moralischen Wahrheiten ist ebenso aussichtslos, wie er schnell unappetitlich zu werden pflegt. Es herrscht dementsprechend ein eklatanter Mangel an einigermassen brauchbaren Gewissheiten und es ist auch nicht absehbar, woher diese denn bezogen werden könnten.

Philosophisch hat man sich in vergleichbaren Situationen eines Mangels an durchsetzungsfähigen Gewissheiten seit der Aufklärung in der Regel damit beholfen, dass man statt mit moralischen Grundsätzen mit Verfahren operierte und genau das scheint auch im Fall der normativen Justierung von Medienkulturen das Mittel der Wahl zu sein. Es geht um ein ziemlich desillusioniertes Sich-Einlassen auf Verfahren der normativen Feinjustierung im Zuge der kulturellen Implementation von neuen medialen Praktiken. Dabei wird eine Dialektik von Beobachtung und Steuerung, die um die Grenzen autopoietischer Selbststeuerung weiss und sich des schal gewordenen Pathos der Heroen der Moderne enthält, in Gang gesetzt und als ein unaufgeregter Prozess permanenter Selbstbeobachtung von Medienkulturen 
installiert, der von der Gewissheit getragen wird, dass noch bislang jede Kultur es geschafft hat, ihrer Medien Herr zu werden und sie ohne grössere Apokalypsen zu zivilisieren. Dass man andererseits auch genötigt ist, Erlösungshoffnungen ad acta zu legen, zivilisiert umgekehrt Theorie. Derartige Ernüchterung tut Not, wenn ziemlich liebgewonnene Matadore wie das autonome Subjekt den medienkulturellen Konditionen nicht mehr standhalten und offenkundig verabschiedet werden müssen. Stattdessen gilt es, mit einem Spektrum von Identitätsmodellen umzugehen und Identität selbst nicht mehr normativ zu verordnen, sondern durch eine pragmatische Performativität zu substituieren. Wenn Identität nicht mehr das Mass aller und damit auch der pädagogischen Dinge ist, dann wird die Form zum Differenzkriterium, das normativ in den Griff bekommen werden sollte.

Der Enkulturalisierungsprozess von Medien ist zweifellos ein Normalisierungsprozess, und wie alle Spielarten der Normalität hat er weniger mit Aufregung und Spannung als mit Mittelmass und Standards zu tun. Trotzdem müssen wir klären, welche Standards wir wollen.

\section{Literatur}

Baacke, Dieter. 1996. "Medienkompetenz - Begrifflichkeit und sozialer Wandel». In Medienkompetenz als Schlüsselbegriff, hrsg. v. Antje von Rein, 112-124. Bad Heilbrunn: Klinkhardt.

Bense, Max. 1965. Ungehorsam der Ideen. Abschließender Traktat über Intelligenz und technische Welt. 3. Aufl., Köln, Berlin: Kiepenheuer \& Witsch 1967.

Brecht, Bertolt. 1932. «Der Rundfunk als Kommunikationsapparat». In Gesammelte Werke in 20 Bänden, Bertolt Brecht, Bd. 18, 133-137. Tsd. Frankfurt a. M.: Suhrkamp 1990, 127-134.

Chomsky, Noam. 1965. Aspects of the Theory of Syntax. Cambridge, Mass.: The MIT Press.

Enzensberger, Hans Magnus. 1970. «Baukasten zu einer Theorie der Medien». Kursbuch 20, März 1970, 159-186.

Gapski, Harald. 2001. Medienkompetenz. Eine Bestandsaufnahme und Vorüberlegungen zu einem systemtheoretischen Rahmenkonzept. Wiesbaden: Westdeutscher Verlag.

Habermas, Jürgen. 1962. Strukturwandel der Öffentlichkeit. Untersuchungen zu einer Kategorie der bürgerlichen Gesellschaft. 5. Auflage, Neuwied/Berlin 1971 Luchterhand.

Habermas, Jürgen. 1981. Theorie des kommunikativen Handelns. Bd. 1: Handlungsrationalität und gesellschaftliche Rationalisierung, Bd. 2: Zur Kritik der funktionalistischen Vernunft. Frankfurt a. M.: Suhrkamp.

Kübler, Hans-Dieter. 1996. «Kompetenz der Kompetenz der Kompetenz ... Anmerkungen zur Lieblingsmetapher der Medienpädagogik». Medien praktisch 2/96 11-15. 